\title{
Effect of spinning workouts on affect
}

\author{
Attila Szabo, Zoltán Gáspár, Nikolett Kiss, and Alexandra Radványi \\ Faculty of Education and Psychology, Institute of Health Promotion and Sport Sciences, Eötvös Loránd University, Budapest, Hungary
}

\begin{abstract}
Background: Numerous physical exercises trigger positive changes in affect after relatively short workouts. Spinning, also known as indoor-cycling, is a very popular form of exercise, especially among women, but its impact on affect have not been examined to date.

Aims: The purpose of the current work was to investigate the possible benefits of spinning on affect in self-controlled and in instructor-led exercise sessions.

Methods: Using baseline measures and pre- to post-exercise design with a psychometrically validated questionnaire, the net effects of spinning (without music) on positive- and negativeaffect were measured in two exercise conditions: (1) self-controlled workout (i.e. without an instructor) and (2) instructor-led workout. After both conditions, 18 women rated the extent which they enjoyed the exercise session on a 10-point Likert scale.

Results: The findings revealed that positive affect increased while negative affect decreased after both workouts. Exerted effort, measured through the heart rate, did not differ between the two conditions. However, participants enjoyed more the instructor-led exercise session than the self-regulated workout (effect size, Cohen's $d=0.93$ ).

Conclusions: This research reveals that spinning improves post-exercise affect, even without music and regardless of instructor's presence. Therefore, it demonstrates the net benefits of this popular exercise on affect.
\end{abstract}

\section{Keywords \\ Endurance training, exercise performance, motivation, physical activity, training}

\author{
History \\ Received 26 August 2014 \\ Revised 27 January 2015 \\ Accepted 4 February 2015
}

\section{Introduction}

It is a consensus that a single bout of exercise improves affect (Anderson \& Brice, 2011; Dasilva et al., 2011; Szabo, 2003a), which is a mental state that reflects how an activity or a particular situation impacts the person (Duncan \& Barrett, 2007). The benefits of a single workout on affect were previously observed in different exercises, including dance aerobics (Rokka et al., 2010), Nordic walking (Stark et al., 2011), running (Hoffman \& Hoffman, 2008; Szabo, 2003a), shadowboxing (Li \& Yin, 2008), swimming (Valentine \& Evans, 2001), taekwondo (Toskovic, 2001), tai chi (Wang et al., 2010), walking (Dasilva et al., 2011), and yoga (Streeter et al., 2010). Spinning, also referred to as exercise biking or indoor cycling, is ranked among top 10 most popular sports among women (Sport England, 2012). However, the effects of spinning on affect were not examined to date.

Most explanations forwarded for the affect-mediating effects of a single bout of exercise are based on exercise volume. Popular physiological models are the endorphin hypothesis (Dunn \& Dishman, 1991; Hoffmann, 1997), the amine hypothesis (Dunn \& Dishman, 1991), and the thermogenic hypothesis (Koltyn, 1997). A more recent explanation

Correspondence: Dr. Attila Szabo, PhD, Associate Professor and Acting Director, Faculty of Education and Psychology, Institute for Health Promotion and Sport Sciences, Eötvös Loránd University, 1117 Budapest, Bogdánfy u. 10, Hungary. E-mail: drattilaszabo@yahoo.com and szabo.attila@ppk.elte.hu relies on a dual-mode model (Ekkekakis \& Acevedo, 2006). Accordingly, changes in affect due to exercise result from the interaction of cognitive appraisal of the exercise situation and the subjective perception of physiological changes during exercise. In spinning, both the presence and characteristics of music, as well as the presence and the motivating power of an instructor may influence the subjective evaluation of the exercise situation, having an impact on the emerging post-exercise affect.

An inverted-U relationship may be observed in the dual model; positive changes in affect emerge below the ventilatory threshold and negative responses above it (Ekkekakis \& Acevedo, 2006). The ventilatory threshold is a point during exercise at which pulmonary ventilation exceeds oxygen consumption. Yet, the second part of the model—pertaining to the intensity of exercise(s) - may be questionable. A number of past investigations have revealed that exercise characteristics have little role in mediating the psychological benefits (Rendi et al., 2008; Szabo, 2003a; Szabo \& Ábrahám, 2012). Research has also demonstrated that an equal-toexercise duration, low- or no-effort activity, like mindful exercises, music, or humor trigger identical, or greater, changes in affect than exercise (Netz \& Lidor, 2003; Szabo, 1988, 2003b, 2005).

More recently, the placebo effect was also linked to the acute changes in affect after exercise (Szabo, 2013). The placebo may mediate both the cognitive appraisal of the exercise situation and the perception of exercise 
characteristics of the dual-mode model. In spinning, for example, expectations associated with the instructor and workout could influence the post-exercise affect, at least in part, via placebo effects. In this context, it should be appreciated that the appraisal and perception of various exercise bouts are affected by past experiences in both healthy and clinical samples (Mason \& Holt, 2012).

In line with the dual-mode model mentioned above, the appraisal of exercise intensity influences affect. Since spinning is a high-intensity exercise, that usually is performed above the ventilatory threshold (López-Miñarro \& Muyor Rodríguez, 2010; Piacentini et al., 2009), according to this model it should generate no positive changes in affect. Spinning is, however, a popular exercise that is usually performed in fitness centres under the direction of an instructor and accompanied by music. In the absence of positive affect, its popularity would be doubtful. However, both the music and the instructor could influence one's affect that may be independent of exercise. Therefore, to assess the "net" effect of a bout of spinning exercise on affect, it is essential to remove the instructor (and the music, because music alone has significant influence on affect; Szabo et al., 2005) from the exercise environment. The removal of both could result in less enjoyable spinning sessions, and lesser positive changes in affect, but can reflect the genuine impact of the exercise.

The current exploratory (two-tailed) research tested: (a) the "net" effect of spinning on affect, (b) the impact of the instructor's presence on changes in affect after spinning, and (c) the level of enjoyment of the spinning session with and without an instructor. The answers to these queries could advance our knowledge about the acute psychological benefits of one of the most popular contemporary fitness and leisure centre exercises. Furthermore, the results could also elucidate whether changes in affect and subjectively perceived level of enjoyment are linked or not to the presence of an instructor.

\section{Method}

\section{Participants}

Clients of a large university-affiliated extramural spinning class were approached and invited to take part in the investigation. As an incentive for participation, a summary of the group results was offered to them. Considering that spinning classes are most popular among women (Reeves, 2012; Sport England, 2012), only female participants were tested. At the beginning, 20 women agreed to participate in the study from a pool of 24 . However, data from one were incomplete and another potential participant did not show up for the second test. Consequently, the final sample consisted of 18 consenting women aged between 19 and 27 years $(M=21.39, S D=2.06)$. All participants were Caucasian university students. They all spoke the same first language (Hungarian) and trained with identical frequency and duration during scheduled spinning classes. The research was conducted in full accordance with institutional as well as international ethical regulations and guidelines (The British Psychological Society, 2010; World Medical Association Declaration of Helsinki, 2008).

\section{Measures}

The Positive Affect Negative Affect Schedule (PANAS Watson et al., 1988) was used for the assessment of affect. We adopted the 20-item psychometrically re-validated Hungarian version of the scale (Gyollai et al., 2011). The scale consists of 10 positive items (i.e. alert, active) and 10 negative items (i.e. nervous, upset). Each item is rated on a 5-point Likert scale ranging from 1 (very slightly or not at all) to 5 (very much). A total score is then obtained for both positive and negative items. The version of the PANAS adopted here has excellent psychometric properties (Gyollai et al., 2011). The internal reliability of the current scale was (Cronbach's $\alpha$ ) 0.82 for positive scale items and 0.83 for negative scale items.

We have also employed a 10-point Likert scale, ranging from 0 (not at all) to 9 (very much) to determine the level of subjective enjoyment of both, the instructor-driven and self-directed work sessions.

For measuring the heart rates (HR) during exercise, we used Polar RS400 HR monitors consisting of chest-band recorder and a wrist-computer receiver. The RS400 receiver unit is compatible with PCs via an IrDA USB adapter. Its recording features include actual and summary measures, adjustable recording rates (at $1,5,15$, or $60 \mathrm{~s}$ ), a memory left indication, individual exercise files, and weekly history. The unit records HR accurately and displays it in beats per minutes and also as percentages (\%) of the maximum HR.

\section{Procedure}

After consenting to participation, volunteers were tested in counter-balanced sessions using a within-participants design. Half of them worked out without an instructor in the first session and the other half with the instructor, who dictated the exercise pace. The instructor was a qualified female aerobic fitness instructor with special accreditation and several years of experience in leading spinning classes. A second test session was performed exactly 1 week later in reverse order. Before exercise, participants completed the PANAS. Subsequently, like during ordinary spinning classes at this and other facilities (i.e. Tilburg University, 2015), we fitted them with a HR monitor using a chest-band electrode and the RS400 receiver. After calibration, participants started spinning for 35 min that also included 2-3 min warm-up and cool-down periods. Apart from usual environmental stimuli, there was no interaction between the class-participants. Since music modifies affect (Szabo et al., 2005), and could act as an extraneous variable, no music was played during the test sessions. Within 5 min after the workout, participants completed the PANAS again and rated the level of enjoyment of the exercise session. HR monitors were collected for determining maximal and average HRs during exercise. The workloads were calculated in terms of percentage of maximal HR.

\section{Data analyses}

We have coded and then recorded the gathered data into an SPSS (Version 17.0, Chicago, IL) file for subsequent statistical analyses. Due to the relatively low sample size, we adopted the less powerful, less sample-size sensitive, and under the situation more reliable Wilcoxon signed-rank non- 
parametric tests. When statistically significant results emerged on the basis of this test, we have also calculated the means- and standard deviations-based effect sizes (Cohen's $d$ ). We used an $\alpha$ level of 0.05 for all statistical tests.

\section{Results}

To check the comparability of baseline measures in the two conditions, we performed Wilcoxon signed-rank tests for positive- and negative-affect before (at baseline) instructorled and self-controlled exercise sessions. These tests were not statistically significant for positive affect, $Z=-1.26$, $p=0.21$, or for negative affect, $Z=-0.70, p=0.94$.

The second set of non-parametric tests were used to test the null hypothesis that no changes in affect occur from preto post-exercise in either instructor-led or self-controlled exercise session. The results, however, revealed that positive affect has increased statistically significantly (Table 1) after both sessions, $Z=-1.94, p=0.05, d=0.39$ (with the instructor) and $Z=-2.09, p=0.03, d=0.41$ (without the instructor), respectively, and negative affect decreased after the two exercise bouts, $Z=-2.25, p=0.02, d=0.38$ (with the instructor) and $Z=-3.20, p=0.001, d=0.91$ (without the instructor), respectively.

Next, to determine whether the changes noted in affect were different in magnitude between the instructor-led and self-controlled exercise sessions, we calculated difference scores by subtracting the post-exercise values from preexercise (baseline) scores. The difference scores for negative and positive affect were then tested with Wilcoxon signedrank tests. No differences in the magnitude of spinninginduced changes were seen in positive- or in negative affect, $Z=-0.28, p=0.78$, and $Z=-0.81, p=0.42$, respectively. Results for measures of affect in the two exercise sessions are summarized in Table 1.

The comparisons of the perceived enjoyment levels of the instructor-led and self-controlled exercise sessions were also compared with a Wilcoxon signed-rank test. The results revealed that participants enjoyed more the instructor-driven session than the self-controlled spinning session, $Z=-3.11$, $p=0.002, d=0.93$ (Table 1). Effort estimates, in terms of the percentage of maximal HR, were calculated for both sessions. The indices of exercise effort were also contrasted between the two sessions by using the Wilcoxon signed-rank test. There were no statistically significant differences in any of the following measures: peak HR, average HR, and percent of the maximal HR (Table 2).

\section{Discussion}

The present findings reveal that a bout of spinning exercise triggers positive changes in affect. This is clearly a net effect that occurs without the invigorating and arousing effects of music and is evident even in the absence of the instructor. Considering that spinning is one of the most popular contemporary exercises (Reeves, 2012; Sport England, 2012), its impact on affect could have important relevance for subjective well-being, as a component of mental health, from both preventive and therapeutic perspectives.

These results may appear controversial in light of the extant knowledge. For example, Ekkekakis \& Petruzzello (1999) reviewed about 200 studies and concluded that highintensity workout has negative impact on affect. In the current research, only crude measures of exercise intensity were taken, but both HRs and percent maximal HR (Table 2) were in the range of vigorous intensity exercises (Mayo Clinic, 2011). Further, the observed improvements in both positive- and negative-affect were seen despite the removal of music (and instructor in one session) from training. It is possible that completion of exercise yields psychological satisfaction that in combination with placebo effects (expectation to feel good after exercise) may trigger changes in affect. It should be highlighted, that while the changes were significant in all instances, the effect sizes were small to moderate except for negative affect in the selfdirected session in which a large effect size was revealed. It is possible that initial disappointment, caused by removal of both music and instructor, caused a pre-exercise increase in negative affect that was counteracted via a positive appraisal of a good workout. Finally, spinning is a phased-interval

Table 2. Means and standard deviation of physiological measures in two conditions.

\begin{tabular}{lcc}
\hline & $\begin{array}{c}\text { Free spinning, } \\
\text { self-controlled } \\
\text { workout }\end{array}$ & $\begin{array}{c}\text { Spinning with } \\
\text { the instructor's } \\
\text { guidance }\end{array}$ \\
\hline Average heart rate & $154.78(11.79)$ & $153.06(14.07)$ \\
Peak (maximal) heart rate & $184.44(8.40)$ & $186.72(6.11)$ \\
Average percent (\%) of & $77.93(5.93)$ & $77.06(6.99)$ \\
maximal heart rate & & \\
\hline
\end{tabular}

No statistically significant differences have emerged between the two exercise sessions.

Table 1. Means and standard deviations of psychological measures in two 35-min spinning exercise conditions.

\begin{tabular}{|c|c|c|}
\hline Positive affect before spinning & $31.33(5.56)$ & $29.44(4.19)$ \\
\hline Negative affect before spinning & $13.06(3.06)$ & $13.61(5.03)$ \\
\hline Negative affect after spinning & $10.89(1.41)$ & $11.83(4.22)$ \\
\hline Difference $(\Delta)$ or change scores ${ }^{\mathrm{a}}$ from pre- to post-exercise & $-256(499)$ & $-239(708)$ \\
\hline
\end{tabular}

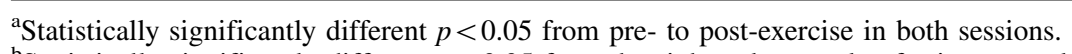

${ }^{\mathrm{b}}$ Statistically significantly different $p<0.05$ from the right column value for instructor-led exercise session. 
exercise in which the person has control over resistance on the spinning bike. These factors could facilitate the generation of positive affect unlike in other forms of high-intensity exercises. Indeed, it was reported that highintensity interval running is more enjoyable than moderateintensity continuous exercise (Bartlett et al., 2011).

The participant-controlled exercise workload was not different from the instructor-led session. There are two explanations for this finding. The first is that memory-based recall of the habitual exercise workload was adopted to set the pace of the workout in the absence of an instructor (and music). The second is that in a co-exercising environment, participants follow the others, which is both motivating and effort-determining, supporting the Köhler motivation-gain effect repeatedly observed in exercise and sport settings (e.g. Irwin et al., 2012).

The robust result revealing that participants enjoyed more the instructor-led workout affords two tentative explanations. The first plausible conjecture is that the mental strategies, such as association and dissociation, were different in the two conditions. Indeed, in the self-directed exercise, participants had to adopt internal association whereas in the alternative session, a continuous attentional focus on the instructor comprised predominantly external association or dissociation (Stevinson \& Biddle, 1998). The key difference between the two mental strategies is that in the former the person pays attention to her/his bodily functions resulting in a high level of awareness, while in the latter the individual pays attention to the external environment, yielding a lower bodily awareness with a delayed perception of fatigue. Further, under instructor's guidance, participants could be more relaxed and not worry about the maintenance of their work pattern. Paying attention to the instructor and harmonizing the movement to her guidance "forces" a mental dissociation. The latter cognitive strategy has been shown to be the most economical and least strenuous in identical modalities of exercise (Neumann \& Brown, 2013), that could be linked to the level of enjoyment of the exercise. A second possible explanation may be that class-participants were conditioned to the instructor-guided exercise classes and felt more relaxed and/or comfortable with the instructor who was seen not only as a facilitator but also as a motivator of the classes.

\section{Limitations of the study}

While exercise intensities, as based on HR measures, were in the high-intensity range in general, in the current study, they were imprecise since intensity was not determined in relation to the participants' fitness level. Further inquiries should define exercise intensity in relation to one's aerobic capacity. In the current study, we were interested in the net effect of spinning and, therefore, removed the music that is played during spinning classes. Further studies should compare affect after spinning in music and no-music situations to demonstrate the external validity of the results and to determine whether music has an additional effect on post-exercise affect. Finally, the current results are restricted to young adults and women only. More work needs to be done to evaluate whether the current results could be replicated with different age groups and men as well.

\section{Conclusion}

The current research contributes three findings: (a) a single bout of spinning exercise triggers positive changes in affect; (b) the benefits in affect emerge in lack of music as well as instructor, therefore, they reflect the net effect of the spinning exercise; (c) the presence of the instructor increases the enjoyment level of the spinning workouts. The implication of the research is that spinning, one of the most popular leisure exercises among women, generates improved affect in healthy young women.

\section{Declaration of interest}

The authors have no conflict of interest to declare. The research did not receive financial support.

\section{References}

Anderson RJ, Brice S. (2011). The mood-enhancing benefits of exercise: Memory biases augment the effect. Psychol Sport Exerc, 12, 79-82.

Bartlett JD, Close GL, MacLaren DP, et al. (2011). High-intensity interval running is perceived to be more enjoyable than moderateintensity continuous exercise: Implications for exercise adherence. J Sport Sci, 29, 547-53.

Dasilva SG, Guidetti L, Buzzachera CF, et al. (2011). Psychophysiological response to self-paced treadmill and overground exercise. Med Sci Sport Exerc, 43, 1114-24.

Duncan S, Barrett LF. (2007). Affect is a form of cognition: A neurobiological analysis. Cogn Emot, 21, 1184-211.

Dunn AL, Dishman RK. (1991). Exercise and the neurobiology of depression. Exerc Sport Sci Rev, 19, 41-98.

Ekkekakis P, Acevedo EO. (2006). Affective responses to acute exercise: Toward a psychobiological dose-response model. In: Acevedo EO, Ekkekakis P, eds. Psychobiology of physical activity. Champaign (IL): Human Kinetics, 91-109.

Ekkekakis P, Petruzzello SJ. (1999). Acute aerobic exercise and affect: Current status, problems and prospects regarding dose-response. Sports Med, 28, 337-74.

Gyollai Á, Simor P, Köteles F, Demetrovics Zs. (2011). Psychometric properties of the Hungarian version of the original and the short form of the Positive and Negative Affect Schedule (PANAS). Neuropsychopharmacol Hungarica, 13, 73-9.

Hoffmann P. (1997). The endorphin hyopothesis. In: Morgan WP, ed. Physical activity and mental health. Washington, DC: Taylor \& Francis, 163-77.

Hoffman MD, Hoffman DR. (2008). Exercisers achieve greater acute exercise-induced mood enhancement than nonexercisers. Arch Phys Med Rehabil 89:358-63.

Irwin BC, Scorniaenchi J, Kerr NL, et al. (2012). Aerobic exercise is promoted when individual performance affects the group: A test of the Köhler motivation gain effect. Ann Behav Med, 44, 151-9.

Koltyn KF. (1997). The thermogenic hypothesis. In: Morgan WP, ed. Physical activity and mental health. Washington, DC: Taylor and Francis, 213-26.

Li G, Yin JC. (2008). The effects of shadowboxing on mood and beta-Ep in still condition of female college students. J Beijing Sport Univ, 31, 357.

López-Miñarro PA, Muyor Rodríguez JM. (2010). Heart rate and overall ratings of perceived exertion during Spinning ${ }^{\circledR}$ cycle indoor session in novice adults. Sci Sports, 25, 238-44.

Mason OJ, Holt R. (2012). Mental health and physical activity interventions: A review of the qualitative literature. J Ment Health, 21, 274-4.

Mayo Clinic. (2011). Exercise intensity: Why it matters, how it's measured; Get the most from your workouts by knowing how to gauge your exercise intensity. Available from: http://www.mayoclinic.com/ health/exercise-intensity/SM00113/NSECTIONGROUP=2 [last accessed 3 Nov 2014].

Netz Y, Lidor R. (2003). Mood alterations in mindful versus aerobic exercise modes. J Psychol, 137, 405-19. 
Neumann DL, Brown J. (2013). The effect of attentional focus strategy on physiological and motor performance during a sit-up exercise. J Psychophysiol, 27, 7-15.

Piacentini MF, Gianfelici A, Faina M, et al. (2009). Evaluation of intensity during an interval Spinning ${ }^{\circledR}$ session: A field study. Sport Sci Health, 5, 29-36.

Reeves A. (2012). Social stratification, gender and sport participation. Soc Res Online, 17, 12. Available from: http://www.socresonline. org.uk/17/2/12.html [last accessed 6 Mar 2015].

Rendi M, Szabo A, Szabó T, et al. (2008). Acute psychological benefits of aerobic exercise: A field study into the effects of exercise characteristics. Psychol Health Med, 13, 180-4.

Rokka S, Mavridis G, Kouli O. (2010). The impact of exercise intensity on mood state of participants in dance aerobics programs. Stud Phys Culture Tourism, 17, 241-5.

Sport England. (2012). Active people survey (APS); Women's participation in sport and physical activity Trends (2005-06 to 2011-12). Available from: http://www.wsff.org.uk/system/1/assets/files/000/000/ 526/526/bdbc729ea/original/WSFF_women\%27s_participationAPS6_ forweb_april13.pdf [last accessed 3 Nov 2014].

Stark R, Schöny W, Kopp M. (2011). Acute effects of a single bout of moderate exercise on psychological well-being in patients with affective disorder during hospital treatment. Neuropsychiatrie: Klinik, Diagnostik, Therapie und Rehabilitation: Organ der Gesellschaft Osterreichischer Nervenarzte und Psychiater, 26, 166-70.

Stevinson CD, Biddle SJH. (1998). Cognitive orientations in marathon running and "hitting the wall". Br J Sports Med, 32, 229-34.

Streeter CC, Whitfield TH, Owen L, et al. (2010). Effects of yoga versus walking on mood, anxiety, and brain GABA levels: A randomized controlled MRS study. J Altern Complement Med, 16, 1145-52.

Szabo A. (2003a). Acute psychological benefits of exercise performed at self-selected workloads: Implications for theory and practice. J Sport Sci Med, 2, 77-87.

Szabo A. (2003b). The acute effects of humor and exercise on mood and anxiety. J Leisure Res, 35, 152-62.
Szabo A. (2013). Acute psychological benefits of exercise: Reconsideration of the placebo effect. J Ment Health, 22, 449-55.

Szabo A, Ábrahám J. (2012). The psychological benefits of recreational running: A field study. Psychol Health Med, 18, 251-61.

Szabo A, Ainsworth SE, Danks PK. (2005). Experimental comparison of the psychological benefits of aerobic exercise, humor, and music. Humor, 18, 235-46.

Szabo A, Meskó A, Caputo A, Gill ÉT. (1998). Examination of exerciseinduced feeling states in four modes of exercise. Int J Sport Psychol, 29, 376-90.

The British Psychological Society. (2010). Code of human research ethics. Available from: http://www.bps.org.uk/sites/default/files/ documents/code_of_human_research_ethics.pdf [last accessed 3 Nov 2014].

Tilburg University. (2015). Spinning and virtual spinning. Available from: https://www.tilburguniversity.edu/about/culture-and-sports/ sports-center/sportsprogrammeandfacilities/spinning/ [last accessed 22 Jan 2015].

Toskovic NN. (2001). Alterations in selected measures of mood with a single bout of dynamic Taekwondo exercise in college-age students. Percept Motor Skills, 92, 1031-8.

Valentine E, Evans C. (2001). The effects of solo singing, choral singing and swimming on mood and physiological indices. Br J Med Psychol, 74, 115-20.

Wang C, Bannuru R, Ramel J, et al. (2010). Tai Chi on psychological well-being: Systematic review and meta-analysis. BMC Complement Alternat Med, 10, 23. Available from: http://www.biomedcentral.com/ 1472-6882/10/23 [last accessed 3 Nov 2014].

Watson D, Clark LA, Tellegen A. (1988). Development and validation of brief measures of positive and negative affect: The PANAS scales. J Person Soc Psychol, 54, 1063-70.

World Medical Association. (2008). World Medical association declaration of Helsinki: Ethical principles for medical research involving human subjects. Available from: http://www.wma.net/en/30publications/10policies/b3/17c.pdf [last accessed 3 Nov 2014]. 